\title{
A nuclear import jam
}

Crystal structures of a transporter with different substrates lead to the design of a pathway-specific nuclear import inhibitor.

Many proteins contain a peptide 'zip code' that targets them to their destination in the cell. Nuclear zip codes are recognized by one of ten import factors, which transport the signal-containing proteins into the nucleus. One of these transporter proteins, Kap $\beta 2$, recognizes the PY nuclear localization signal (NLS), a zip code that is different from the classical short, basic NLS that binds Kap $\alpha-K a p \beta 1$.

After solving the structure of Kap $\beta 2$ bound to a PY-NLS-containing substrate, Yuh Min Chook and colleagues at the University of Texas Southwestern Medical Center at Dallas defined Kap $\beta 2$ signal recognition rules (Lee et al., 2006), and then identified other potential substrates. "We needed to validate these proteins as Kap $\beta 2$ substrates, especially in vivo, but this is a cumbersome task," says Chook. "It would be helpful if we had an inhibitor [of
Kap $\beta 2]$, but there were none."

Given sufficient manpower and funding, a chemical screen can be a way to find an inhibitor, but this option is not always available to a small, young lab like Chook's. Instead, they found a way to design a peptide inhibitor when they solved the structure of another PYNLS-containing substrate bound to Kap $\beta 2$ (Cansizoglu et al., 2007). A comparison of this complex with the previously solved one revealed that the two signals are structurally different but converge at three spots. These epitopes each bind Kap $\beta 2$ quasi-independently and with varying affinities, and most importantly, the binding hot spots in the two NLSs are asymmetrically located.

Armed with this information, Chook and colleagues saw an opportunity to design a tight binder to jam up Kap $\beta 2$, and thus inhibit nuclear import via this pathway. Their peptide, M9M, is a chimera of the identified asymmetric hot spots. In HeLa cells, M9M blocks nuclear import of several Kap $\beta 2$ substrates because of its tighter binding. "It's so tight that it's not a signal anymore because it can't get released in the nucleus, and so it becomes an inhibitor," explains Chook.

As well as using M9M to validate potential Kap $\beta 2$ substrates, the researchers aim to map the potential redundancy in import networks. In the future, Chook hopes to make a chemical inhibitor but underscores that "it will be very hard to find one small molecule that could block NLS recognition as well as our peptide, but you can presumably identify multiple compounds that bind to the different epitopes and tether them together."

Irene Kaganman

\section{RESEARCH PAPERS}

Cansizoglu, A.E. et al. Structure-based design of a pathway-specific nuclear import inhibitor. Nat. Struct. Mol. Biol. 14, 452-454 (2007).

Lee, B.J. et al. Rules for nuclear localization sequence recognition by Karyopherin $\beta 2$. Cell 126, 543-558 (2006). 